\title{
Towards an Artificial Pancreas: Software Architectural Model and Implementation for Personalized Insulin Administration
}

\author{
Mari Gunleiksrud Jensen \\ University of South East \\ Norway \\ 130962@usn.no
}

\author{
Radmila Juric \\ University of South East \\ Norway \\ rju@usn.no
}

\author{
Karoline Moholth \\ McClenaghan \\ University of South East \\ Norway \\ karolinem@usn.no
}

\author{
Gordana Blagojevic \\ Zagorac \\ Faculty of Medicine \\ University of Rijeka \\ Croatia \\ gordana.blagojevic@uniri.hr
}

\begin{abstract}
Research on an Artificial Pancreas has gained its momentum and focused on the processing of clinical data for continuous insulin administration. However, the overall research is rather sketchy, fragmented and not very well coordinated. In this paper, we propose an architectural model for creating software intensive environments, which address deficiencies of current solutions for insulin infusion. A new way of defining which data should be collected and which types of computations should be performed with the data is important if we wish to come close to the functioning of a natural pancreas. An excerpt of the proposed software architecture has been deployed using Watson Analytics and performed upon a selection of data collected from sensors, individual patient's input and persistent patient records.
\end{abstract}

\section{Introduction}

According to the Norwegian "National Diabetes Plan 2017-2020" [16] there are approximately 245000 persons diagnosed with diabetes in Norway, of whom 28000 have DMT1. Each year there are another 300 children (age $<15$ years) diagnosed with DMT1. In Norway, the annual increase in number of newly diagnosed diabetics is less pronounced, though the total number of persons living with diabetes (DM) continues to grow. In 2014, the World Health Organization reported that there was an estimated worldwide number of 422 million adults living with DM [31], and the estimated total monetary cost of diabetes is more than 827 billion USD per annum.

$\mathrm{DM}$ is a chronic non-communicable disease that causes the patient pancreas to stop producing insulin [1]. As an autoimmune response to some unknown factor: the beta-cells of the pancreas are destroyed and ultimately unable to produce enough, if any, insulin. If left untreated, the Blood Glucose (BG) levels continue to increase, the metabolic processes deteriorate, and the patient shows symptoms of hyperglycaemia, which may cause coma and/or death [14, 22]. During the last 30 years there has been tremendous progress in the research and development of technological aids for diabetes management. Today, we talk about hybrid closed-loop system, which sense a patient's BG level, calculate and administer a basal, or bolus, dose of insulin. They consist of a BG measuring device, a continuous glucose-monitoring unit (a control unit), and an insulin infusion unit. Open-loop insulin infusion systems are different because the control unit, often placed within the insulin infusion device, is dependent on the patient manual input to calculate an insulin dose. The patient must approve the dose or adjust it according to their own calculations. An Artificial Pancreas (AP), which is expected to be a closed-loop system, aims to perform these tasks with little or no manual user input.

In this paper, we analyse research ideas for the development of an AP and systemise problems found in the relevant literature. We propose a new way of looking at the development of an AP by revisiting data sets and computations found in current insulin administrations. The outcome is interesting. The data sets currently processed in open/closed loop insulin infusion systems, are not sufficiently rich and broad to be compared with the data our natural pancreas uses in insulin administration. Furthermore, the processing of the data appears to be insulin pump manufacturer specific and does not allow for variations in the way we collect and process data. Therefore, we advocate changes in both: data types we collect and the type of computations we can perform upon the data.

The paper is organized as follows. In section 2 we overview a selection of research papers which either influenced our research or address some of the aspects of insulin administration, which could mimic the 
functioning of a natural pancreas. Section 3 itemises problems we detected in the literature, which focuses on various aspects of creating an AP. In section 4 we analyse additional problems which appear within current insulin administration systems and in Section 5 we offer our proposal which addresses these problems. The proposal consists of (i) a new data model and (ii) a software architecture, for creating a software intensive environment for insulin administration. We expect that the solution would closely mimic the functionality of a natural pancreas. In section 6 we conclude by outlining what the next step in the development of an AP could be.

\section{Related Work}

\subsection{Evolution of Insulin Administration}

The administration of insulin entails a basal rate, to ensure the constant presence of insulin [8], and a bolus amount injected. It compensates for spikes in BG levels, due to food intake or unexpected hyperglycaemia [6]. During the last 30 years, this process changed from an analogue procedure to using increasingly advanced technologies. In 2004, Siebenhofer et al [21] performed a meta-analysis of 27 randomized control trials to compare the effect of treating DMT1 patients either with short-acting insulin analogues or with regular insulin (non-analogue). The treatments were administered through continuous subcutaneous insulin infusion (CSII) or as intensified insulin therapy (IIT). The results suggest that the rate of hypo-glycemic episodes was NOT reduced by using analogues (compared with non-analogue), but the CSII therapy provides a small, yet statistically significant, improvement in glycemic control compared to that of IIT. In 2011 Wang et al [30] describe the "glucosemeal-insulin dynamics" as a "traditional batch process" and explain how run-to-run (R2R) algorithms are used to calculate the bolus amount of insulin to administer. By 2014, we had a complete and well written description of what AP is in [19] and in 2015, Barnard et al [3] have performed a systematic review of available research on the psychosocial and psychological measures suitable for use in the research of future AP's. They found very little evidence that research, related to AP, includes these measures.

\subsection{Open-Loop Systems}

In addition to using the improved "basic" Blood Glucose Measuring (BGM) devices, patients can now track their interstitial glucose levels using a Continuous Glucose Monitoring (CGM) device. Current state of art includes features that enable both devices to connect to and/or transmit the measurements to an insulin pump. The pump sends these inputs to some pre-programmed algorithms and advises the patient to adjust their insulin delivery. It may suggest a calculated insulin dose or even provide an alarm if the BG level is outside, or nearing, a preset safe range of glucose concentration [15]. The patient must, however, always perform their own calculation and adjust or administer a dose according to their own individual experience and knowledge [29]. The current open-loop systems are dependent on these inputs, to ensure safe and reliable dosages being infused with the correct time interval [6].

\subsection{Closed-Loop Insulin Infusion Systems}

Closed-loop systems are often termed as an AP. [26]. The authors claim that the end goal is to create a real-time embedded system that requires little to no management from the patient, yet function at the highest level of accuracy, safety and reliability [1416]. They usually consist of three units: a glucose monitoring unit, an insulin infusion unit and a control unit [7]. The AP could, however, also include the infusion of glucagon, to compensate for decreasing $\mathrm{BG}$ in the way a real pancreas would [9].

\subsection{Closing the Loop with Algorithms}

Historically, research has focused on creating devices for either glucose monitoring or insulin infusion [7]. To create and close the loop, the control unit contains algorithms within the insulin infusion device, or sometimes becomes a part of a separate control device managed by the user/patient $[7,27,28$, 32]. The algorithms use collected data (i.e. BG) as an input to calculate a dose of insulin, to be administered through the insulin infusion unit

One of the frequently mentioned algorithm form [30] is based on Model-Predictive-Control (MPC) and Iterative Learning Control (ILC) to perform glycaemic control. It does not require user intervention and might be applicable to children and adolescents. The Turksoy et al form [27] present a different approach: they created a multimodule AP system, which includes adaptive algorithms for handling unexpected changes in physiological variables received from wearable devices. Therefore, they note that exercise may affect BG concentrations. The algorithm from [28], used for meal-detection, is based on CGM measurements and predicts almost all tested meals in their experiments.

Toffanin et al [25] propose an adaptive MPC algorithm based on an R2R approach, to potentially "capture intra- and inter-day glucose variability...". It uses the data from a CGM and calculates a basal dose during night and a "carbohydrate-to-insulin ratio" (CR) in the day. Boiroux et al [6] propose a method to track the postprandial glucose dynamics and insulin 
sensitivity. Using a virtual model, they created a calculator for basal and bolus doses. It is dependent on patients entering their meals to calculate the correct bolus dose. They used a switching strategy between meal and insulin sensitivity estimation to allow for tracking the correct insulin sensitivity.

Samadi et al [20] showed the algorithm for detecting meals and estimating their size in the form of carbohydrate amount. It is based on "the interpretation of qualitative representation of CGM signals" and integrates wavelet filter, qualitative representation and fuzzy logic.

The algorithm from Ortmann et al [17], is based on MPC and considers periodic insulin sensitivity (IS), which changes with a patient's circadian rhythm, to improve BG control. They use machine learning (ML) to predict the effect of the IS. Bamgbose et al [2] propose a simulation framework, also based on ML techniques, for an integrated control system of administering bolus. The predictive controller uses the patient's medical records for its calculations. The only clinical data processed are insulin and BG levels.

Keith-Hynes et al [12] propose a "Diabetes Assistant" (DiAs) as an AP research platform. They perform clinical studies of AP's on portable devices and smartphones. It will be interesting to see if their ideas materialise. Lazaro et al [13] propose a closedloop AP system implemented on a mobile device by integrating an insulin pump, a GCM, and other sensors, with various software components, which should have a common goal of controlling BG levels. It is important to note that there are safety implications, issues of data accuracy and the reliability of data input via user interface [5]. The increased risk of life-threatening complications for those living with DMT1, must be considered when developing an AP which assumes the roles of a human organ.

\section{The Problem}

The development of an embedded real-time system, which mimics the biological functions of a human organ, has many challenges. Current research on an AP seems to be fragmented and scattered around various devices and software embedded within them. Although each of them contributes towards an AP, this fragmentation makes it difficult to discover

(a) where and how to streamline further improvements in the development of an AP;

(b) where the obstacles of developing a full-scale AP actually lie;

(c) if we could create a software intensive solution which replaces the functioning of our natural organ".
In trying to address (a)-(c) we summarise problems from the literature into P1-P5.

Problem P1: Which clinical data should be collected and manipulated?

There is no consensus in AP research which answers problem P1. Control units depend on clinical measurements as input for their control algorithms. Manufacturers of these devices claim that they focus on the data, which ensure "correct insulin administration". Has anyone analysed the full scale of data spectrum used in the functioning of a natural organ? Do current solutions for AP take them all into account? In just one simple example, where the glucose concentration of interstitial fluid of the subcutaneous tissue, is juxtaposed to glucose concentration of the intravenous blood, we do not have equal values. Do CGM devices compensate for that lag? We found devices that may be placed intra peritoneum, to achieve direct access to blood glucose, but how likely is it that this will become a mainstream solution for an AP?

Problem P2: Which non-clinical data could be relevant for creating an AP?

The human body and our metabolism are affected by many factors. When calculating an insulin dosage, non-clinical data may prove to be valuable data inputs. These include subjective/personal data, environmental conditions, ambient temperature, psychosomatic issues and the body's ability to process food. No published work exists which considers these factors as part of a "holistic algorithm" for controlling insulin infusion.

Problem P3: How does the Insulin Sensitivity Factor affect the functioning of an AP?

Insulin sensitivity (IS) is the definition of how quickly and easily the body reacts to the effects of insulin (both endo- and exogenous). As an AP may include continuous monitoring of $\mathrm{BG}$, we question if this factor is needed as an input for the algorithms, or will it become redundant for an AP?

Problem P4: How would Active Insulin affect bolus algorithms in an AP?

We assume that an AP will work by continuously administering small, well-calculated, doses of insulin, without inputs from the user. Will it therefore need to compensate for this active insulin according to its own calculations?

Problem P5.1 - Current data processing and control algorithms do not address most of the problems from P1-P4 and should be revisited.

The data in the current AP solutions is very often processed by devices, which collect them and administer insulin. The algorithms are embedded within devices, but in the era of Big Data technologies, embedding software solutions into devices does not 
look promising. An AP needs continuous computations, which cannot be hindered by the preprocessed data with embedded software. Would embedded software allow changes in the way AP data is collected and processed?

Problem P5.2 - Should AP enable a greater patient centric treatment regime?

The problem of computing a correct insulin treatment regime should be resolved at the patient level and should not be dependent on a) scheduled visits to a specialized healthcare professional and $b$ ) rules prescribed by the embedded software in AP devices. An AP should work autonomously and independently, by providing patients with advice and warnings, for adjustments to the treatment regime. Could we achieve this through our computations?

\section{Example of Insulin Administration}

Before we try to address problems P1-P5 we

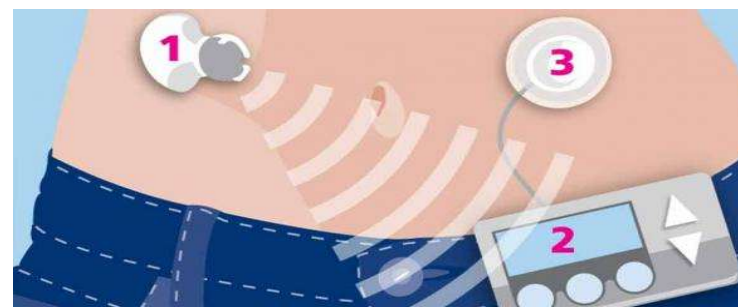

Figure 1 - Open-Looped or Hybrid Insulin Administration System

analyse one of the hybrid insulin administration systems available in Norway [2]. Figure 1 is a general visualization of such an infusion system.

The CGM device (1) is attached with a small sensor needle placed in the subcutaneous tissue. This measures the glucose concentration every 5 minutes and transfers the results to the control unit within the insulin infusion device (2). The control unit is the most advanced of the sub-systems and contains settings and algorithms which define the functionality of the entire system. Upon receiving the sensory data, the control unit performs various calculations and provides auditory and visual warnings, when necessary. This is the unit which controls and monitors the infusion of insulin through the infusion site (3). Being an openloop system means that all functions within the control unit rely on settings and inputs provided by the user.

The "Medtronic CareLink Personal" is a web application which allows uploading the insulin administration data and generating reports from their clinical diabetes related data. These reports are very detailed, and patients need a certain degree of technical and analytical skills to understand them. The grouped reports are detailed and cluttered, which makes them difficult to interpret. The single reports consist of the same six columns, regardless of which report is selected, and are useful only when searching for very specific data and results.

We ran reports on progress and BG trends, glucose measurements correlated to meals, insulin calculations and administrations and glucose measurements and flagged events. However, our interest was in the data set generated by this hybrid open loop system, which was downloadable as a .csv-file via the webapplication and viewed by MS Office Excel. Column headings of datatypes collected by the control unit are BWZ of calculation (E), goal high BG $(\mathrm{mmol} / \mathrm{L})$, goal low BG (mmol/), carbohydrate data input (gram) BWZ BG data input (mmol/L), BWZ correctional calculation (E) and food calculation (E), where BWZ stands for Bolus Wizard Data. There are also numerous data setting which cannot be discussed due to space limitation.

\subsection{Manual Analysis of the Dataset}

The manual analysis of the data set revealed interesting results. The structure of the spreadsheet and its columns explains the meaning of the data within it. However, the spreadsheet contains both raw and processed data, and it remained unknown to us how that data was processed. It was obvious that prior computation must have been performed on some of the data, as they contain attributes as well as values. Therefore, the data have different levels of values, which automatically signalled that we might not be able to perform further processing of the dataset. This becomes an obstacle in performing any other analytical tasks, except for the ones already determined by the manufacturer of the control unit. This is clearly a proprietary solution, set to work in restricted environments, and by only a selected number of stakeholders and users. It is not a reusable, nor available model for data collection or different processing and decision making we may need in future. This was an unexpected disappointment: this relatively widely used software solution in Norway, is probably outdated in today's climate of increasingly advanced data processing technology, particularly in Big Data environments.

\subsection{Applying WA to the Dataset}

Our initial idea of using the Big Data technologies upon sensory generated data, which could help in insulin administration, was tested by applying Watson Analytics (WA) on this dataset. We faced numerous problems: our data set was of only $9 \%$ quality, vetted by WA; column headers interpreted by WA made no sense; several columns related to the time stamps appeared, and the discovery set defined by WA was 
impossible to interpret. The most predominant value in our data set found by WA was BLANK. Obviously, we could not process the data set by WA.

Though we expect WA to accept an untouched dataset, and enable us to run analysis directly, this was not the case. Most of the columns contained Norwegian words, which were changed to unrecognizable and highly unsuitable names, making it impossible to create sensible discovery sets. Though the set was quite large (over 13.000 rows) many of the cells were empty, decreasing the quality score. The fact that the so-called "raw data" is already semiprocessed and stored as values within one single column of the original file, seem to "confuse" WA, which makes analysis even more troublesome.

\section{The Proposal}

Section 3 and 4 highlighted that we have to address two important problems when developing an AP.

The first focuses on the creation of a new data model, which would address P1-P4 from section 3 and would be sufficient for performing computations, which mimic functionalities of a natural pancreas. We found no publications, which explore the world of the natural pancreas first and then mirror it to the world of data collection from our body. It is not sufficient to base calculations for insulin administration solely on BG and SG readings and a small selection of clinical data closely related to glucose/insulin levels and the food patients consume.

The second focuses on P5.1 and P5.2. and the choices of insulin infusion devices. They process collected data, but the processing is proprietary of the device's manufacturer(s). This makes any changes to the data devices collect and process, impossible. Sensors around our bodies would generate an excessive amount of data, and their processing with Big Data technologies might be inevitable in the modelling of the functionality of an AP. In the current climate, the data we collect, and have access to, are not suitable for further processing, except within software solutions provided by device manufacturers. Therefore, we propose:

a) A new data model for creating computations for an AP and addressing P1-P4;

b) A Software Architectural model, which uses the data from a) and addresses P5.1 and P5.2.;

c) An excerpt of the implementation of our Software Architectural model from b) with IBM WA. This can build a long-term strategy of personalized insulin administration by patients.

\subsection{Data Model for an AP}

Table 1 - The proposed data model

\begin{tabular}{|l|l|l|}
\hline Diabetic Specific Data & $\begin{array}{l}\text { General } \\
\text { Clinical Data }\end{array}$ & $\begin{array}{l}\text { Subjective, Non- } \\
\text { clinical data }\end{array}$ \\
\hline BG Current & Cortisol & Psychosomatic \\
Low BG Target & Adrenalin & issues \\
High BG Target & Serotonin & Sleeping disorders \\
BG Range & Dopamine & Circadian rhythm \\
Default High BG Value & Liver impact & Subjective feelings \\
SG Current & (metabolizing & Anxiety \\
SG Range & glycogen into & Fear \\
SG High Alert & glucose) & Depressive \\
Insulin-to-Carb Ratio & Menstrual Cycle & thoughts \\
Insulin sensitivity factor & Body Fat & Taking anti- \\
Active insulin time & Amount of & depressant \\
Bolus Speed Standard & muscles & Taking \\
Bolus Speed Quick & Body & Contraceptives \\
Bolus Programming & temperature & Time of day/night \\
Increment & Common Cold & Location \\
Insulin corr. Estimate & Flu & Weather \\
Fluid Delivered/Stroke & Biome in gut & Ambient \\
& & temperature \\
& \multicolumn{2}{|l}{ Current activities } \\
& & Exercise \\
\hline
\end{tabular}

Table 1 shows the proposed data model from a). The left column contains known Diabetic Specific Clinical Data, which we expect to be either collected or calculated. Some of the data from this column are settings for enabling AP calculations, which could either be prescribed in advance, or dynamically changed by the patient and/or AP. The middle column in Table 1 contains clinical data which may influence the administration of insulin but are not directly related to $\mathrm{BG}$ measurements. The right column contains patient personal/ subjective data which characterises the environment of an individual patient. These three distinctive columns are not created by accident. The data from the left column are mostly present in insulin infusion systems based on devices, which collect, and process sensor generated data (BG and SG). The data from the middle column are chosen to add more semantics to diabetes specific clinical data: because they might affect insulin secretion. The data from the right column are personalised observations, feelings and perceptions patients may have about themselves, which may also affect the way insulin is administered. Each column contains data collected and processed differently, but the data across columns are interwoven and influence each other even outside these column barriers. In current commercial solutions of insulin infusions, only data from the left column are used and the semantics stored in the middle and right column are largely ignored.

The rationale behind and description of the data model from Table 1 are given in the paragraphs below. 
BG versus SG. The best approach to insulin infusion would be to directly measure BG with

intravenous implantable devices to avoid wrong calculations of BG from SG readings. Unfortunately, only subcutaneous and microdialysis studies have yielded commercial products. All sensors are enzymebased and boronic acid derivatives, as enzyme-freebased assays, might not be in clinical use. Also, environment temperature and $\mathrm{pH}$ of intestinal fluids can lead to wrong readings of SG and consequently to wrong calculations of BG. Furthermore, there is a delay between BG and SG changes, which can be between 5 and $15 \mathrm{~min}$, and thus life-threatening, because the patient could already be in hypoglycaemia with still normal SG readings. One would think that if BG measurements cannot be achieved instantly, then we should set alerts for the high and low SG levels. However, they must be highly personalized. We have to measure BG (with prick test) and SG (with sensor) at the same time and define/set their relationship for each patient individually. Obviously, in a fully functioning AP these relationships are prerequisites, if we still wish to use SG levels in BG calculations.

Insulin Sensitivity and Insulin Correction Factors. The Insulin sensitivity factor is the drop in BG level caused by each unit of insulin administered. For this factor BG levels are in $\mathrm{mg} / \mathrm{dL}$ and it can be calculated by dividing 1800 (rapid insulin) with the number of daily doses of insulin. Situations that cause the insulin to carb ratio to rise will cause the insulin sensitivity factor to drop, i.e. the insulin to carb ratio will rise because of the drop of insulin sensitivity and thus the insulin correction factor is calculated similarly: 100 / TDD (total daily doses) in mmol/L. Obviously, these two factors are heavily interwoven.

Insulin Sensitivity and Insulin to Carb Ratio. Further analysis of insulin sensitivity factors takes us to insulin to carb ratio. We have to collect a new set of data if we wish to mimic the functioning of a natural pancreas. The data from the second and third column in Table 1 are created from 1) - 7) below. This is very important, because the calculations of insulin to carb ratios vary and depend on other types of data not identified in the left column in Table 1.

1) The insulin to carb ratio should be higher in the mornings because of the Down Phenomena. Our body produces more hormones (growth hormone, cortisol and adrenalin) that trigger the liver to metabolize glycogen to glucose and pump it into the blood.

2) When hypoglycemia occurs during the night, due to the Somogyi Effect, our body goes into panic mode and secretes hormones like glucagon and adrenaline, which does the same: trigger the liver to metabolize glycogen into glucose and pump it into the blood.

3) The insulin to carb ratio changes during the menstrual cycle. In the follicular phase insulin sensitivity increases because of increased FSH levels and thus the insulin to carb ratio should be lower. In the early luteal phase insulin sensitivity decreases because of the increase of progesterone levels and the insulin to carb ratio should be higher. The use of hormone-based contraceptives also affects the insulin to carb ratio.

4) During illness (especially viruses) sensitivity to insulin is lower and the insulin to carb ratio should be higher.

5) Stressful situations (with high cortisol levels) require increasing the insulin to carb ratio. Cortisol causes decreased insulin sensitivity and triggers the liver to release more glucose into the blood.

6) The insulin to carb ratio should be lower during exercise and when we are in the honeymoon mode because (i) the sensitivity to insulin is higher and (ii) muscles can uptake glucose without insulin during contraction.

7) Body fat changes the insulin to carb ratio: the more fat the higher insulin to carb ratio should be. We should measure the amount of fat and muscles because the insulin to carb ratio will not necessarily be the same for the same weight of person.

Insulin Sensitivity, Psychological Factors and Neurotransmitters. It is important to note that insulin to carb and insulin sensitivity factor variations, affected by psychology state, exercise, fear, happiness and similar could be "detected" either by patient subjective feelings or by measuring levels of different hormones and neurotransmitters. The data from the middle and right columns cannot be avoided and their presence is further justified in a-d.:

a. Acute stress must be measured through adrenalin levels, but as of today, it is still not possible to get continuous measurement of adrenalin. Chronic stress should be measured by cortisol levels, although if the person is exposed to stress for a very long time, cortisol levels can be low due to adrenal fatigue. Continuous measurement of cortisol is done by subcutaneous microdialysis [4] and research on AP should address it.

b. Serotonin influences and is influenced by insulin release. It stimulates the liver to produce more glucose. Antidepressants, which mostly work on the principle that they change serotonin levels, also affect insulin secretion. A rise in serotonin may be the key why we need less insulin when we are in the honeymoon mood. [18] states, that a lack of serotonin in the pancreas causes diabetes. Serotonin does raise blood glucose levels, because it forces the liver to 
produce more sugar, but this effect is stronger than stimulation of insulin secretion.

c. The neurotransmitter dopamine raises glucose: it stimulates the liver to pump glucose into the blood.

d. Gut biota shows that mice with sterile guts are more prone to diabetes and therefore we should look at the role it may have in the creation of AP.

To summarise, the content of Table 1 shows a minimum number of data to be collected if we wish to create computations, which mimic the functionality of a natural pancreas. They may be measured through sensors, calculated upon their readings, entered by patients and doctors, and retrieved from any persistence, including patient healthcare data. Explanations on how to collect the data and perform calculations, is outside the scope of this paper.

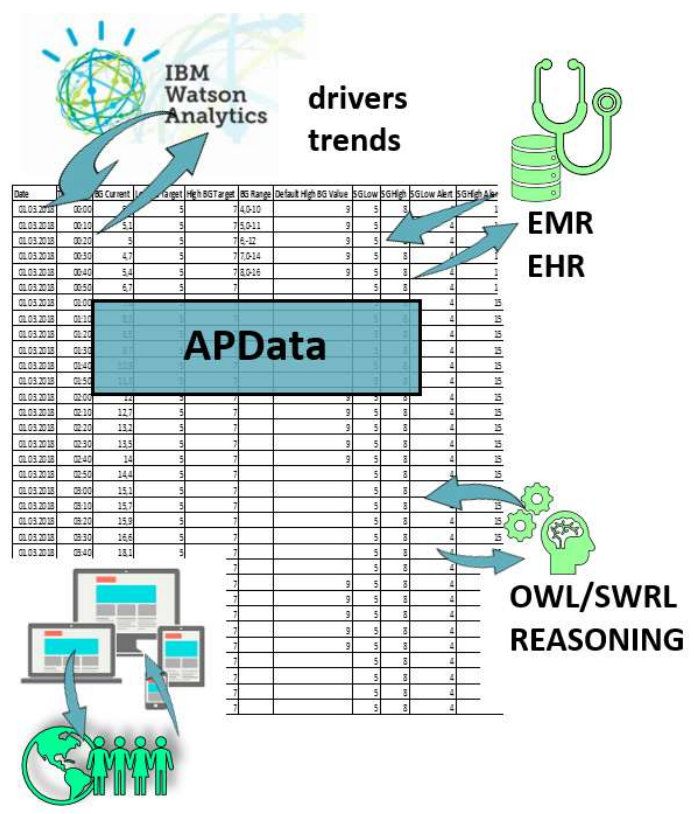

\section{Figure 2 - The Scenario for the Proposed Data Model}

\subsection{Software Architectural Model}

Figure 2 places the proposed data model, from the previous section, within the scenario in which data processing is carried out according to functionalities typical of a natural pancreas. Therefore, patient medical, and/or clinical and/or healthcare records (EMR/HER) are being manipulated and supported by the content of the APData model. The semantics, behind the data placed in Table 1 should be manipulated through the reasoning upon SWRL enabled OWL ontologies. Glucose drivers and trends could be obtained at any time from the APData, using WA. However, these three computations indicated in Figure 2 do not exist in isolation. They are interwoven because they share all data repositories and use the semantics stored in them, as a natural pancreas would do. The idea of manipulating the data model from Table 1, illustrated in Figure 2 is transformed into the software architectural model in Figure 3, which generates a Software Application for an AP.

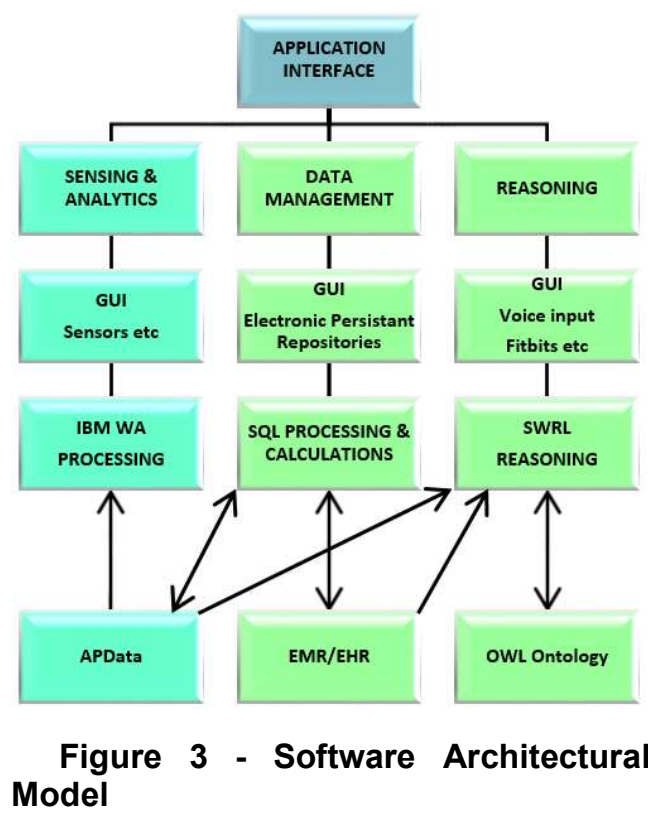

Figure 3 shows software architectural components which enable computations upon the data model from Table 1, which could mimic functionalities of a natural pancreas. The bottom set of software components are different types of data storage to enable these computations. We can run WA upon the APData, manage persistent clinical data and patient healthcare records and reason upon the semantics we store in the data model from Table 1 in OWL ontology. Three different software technologies are used:

a) WA for creating predictions and finding trends upon the patient's management of BG;

b) Transactional processing with the support of $\mathrm{SQL}$, for performing calculations for insulin infusion, and updating persistent data and EMR;

c) Semantic Web Technologies for performing reasoning upon SWRL enabled OWL ontologies for defining and manipulating the correlation between and meaning of data stored in APData/ Table 1. 
Software applications generated from Figure 3 can be deployed across various component based integrated development environments and Android (53). We can process data with traditional transactional processing and reasoning upon OWL ontologies in parallel $[11,23,24]$ and run the analytics by WA upon APData. Detailed computations for the Data Management and Reasoning parts of Figure 3 are outside the scope of this paper but can be found in our earlier publications [10, 23].

The semantic overlapping between data described in Table 1 is managed by OWL concepts. The result of reasoning with SWRL upon OWL will influence calculations of insulin infusion and result in updating persistent data, including APData, if necessary. Therefore, Figure 3 has multiple roles: (a) It shows architectural components which must be deployed to mimic the functionalities of an AP and (b) it defines variations in types of computations with data sharing amongst them. Sensors, which collect data, could not be shown in any software architectural model, but the data they generate have their place in sections of the APData model from Table 1. Sensors we will use in future should only collect data, which means that the architecture from Figure 3 is not insulin infusion device specific.

\subsection{Running WA upon APData}

Our APData content was created according to the data model from Table 1. The excerpts from its content were copied from the data generated by the insulin pump used in our experiment. These pumps generate only a fraction of the data needed for the APData model and therefore all subjective and nonclinical data were collected/inserted through user input. We had the data for 24-hour time/stamp and the field(s), in which we had no available data, were left empty.

After uploading the APData into WA we ran numerous experiments and explored all possible options given by WA to define trends and insights on the data stored in APData. Initially, we did not wish to create any particular question to which we may have wanted to obtain the answer using WA. The purpose was to find out and assess the value of data analytics offered by WA. Due to space restriction, we cannot show the scale of our experiments and results, but would mention results, which surprised us.

Naturally, we were interested to see weather WA would define main "drivers" for the BG data and their strengths. This would give us an indication of whether there were any co-relations between data stored in different columns of Table 1. We were not interested in results of specific calculations relevant to insulin administration. We wanted to know how WA would manipulate the semantics of data stored outside the left column. Figure 4 shows the main drivers for current BG levels and their strengths and places "Current Activity", "Location" and "Body Temperature" as drivers of $58 \%, 57 \%$ and $53 \%$ strength respectively.

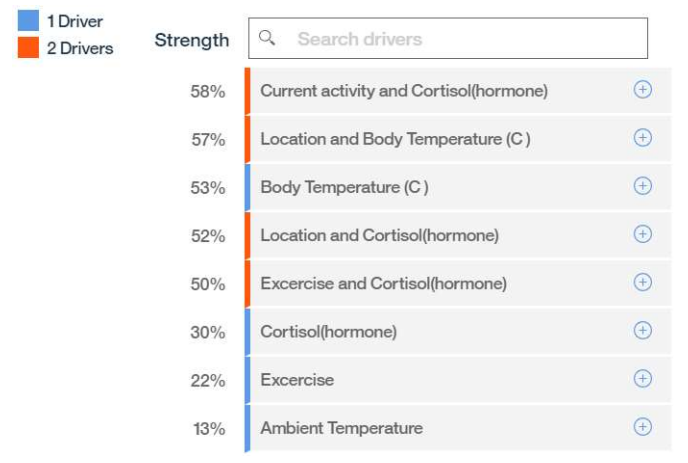

Figure 4 - Main Drivers for Current BG

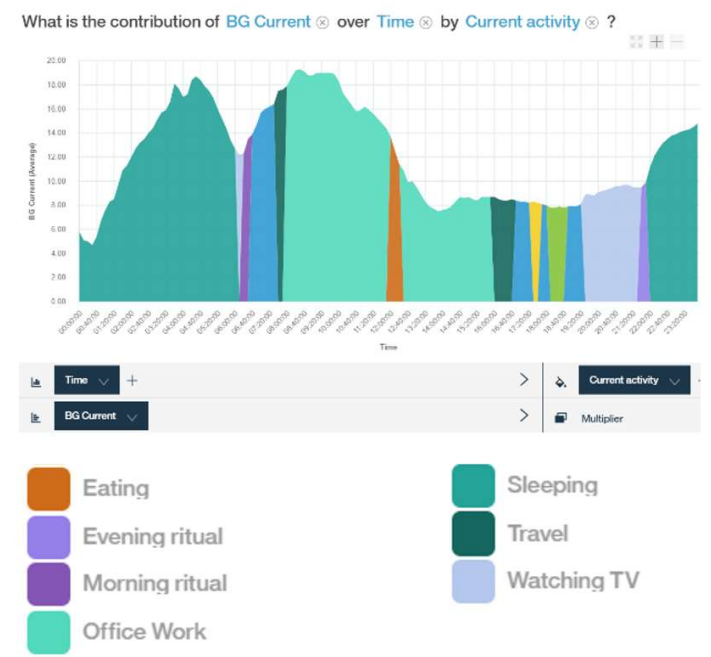

\section{Figure 5 - Contribution of BG Current over Time by Current Activity}

Figure 5 shows the contribution of BG current over TIME and CURRENT ACTIVITY and shows which activities impact current BG levels: from child care, cleaning/cooking, to sleeping/Watching TV!

WA also allowed creating our questions and provided the starting point for exploring the content of APData further. The use of WA proved to be user friendly and did not require special technical skills for running the experiments. Therefore, it can be used for long-term analysis of the changes in BG levels and insulin administration per patient, and include the impact of factors outside clinical data on BG levels and consequently on insulin administration. 


\section{Conclusions}

The initial motivation for this research was to process sensor generated data created by insulin pumps, using Big Data technologies. Sensor generated data, continuously collected by insulin pumps, could be instantly processed by WA and advice patients on BG management on an ad-hoc basis. This initial idea seemed realistic at the beginning of our research.

However, after inspecting the functioning of one insulin pump, and looking at all visualization provided by the software application which supports the pump, it was obvious that the data collected through the insulin infusion system could not be processed further by Big Data technologies. Furthermore, the data collected by current insulin infusion systems are not sufficient to mimic the calculations of insulin infusion typical for a natural pancreas. We needed both: a new set of data and a new way of processing it.

We could not find any publication that could address the problem of the definition of a new data set and its processing for an AP. Therefore, this research is an attempt towards creating a full-scale software solution for an AP. In computations, we have to come closer to the functioning of our natural organ: we created a new dataset in Table 1, and a new set of computations that share data involved in them for insulin administration in Figures 2 and 3. In this paper, we show only one part of our implementation, which contained WA. This is a suitable environment for an ad-hoc testing of the content of big data sets and is available as a free platform for academic research.

There are two outcomes of this research. Firstly, we have paved a different way towards an AP because:

- We propose a software model that utilizes three different types of computations.

- The software architectural model is reusable and may be used for any other organ that needs continuous medication/treatment.

- WA upon the new data model is efficient, but any other Big Data technology could do the same. Our model is not WA specific.

- Software applications which compute OWL reasoning and SQL calculations co-exist and have been implemented in our previous research [23]

- Any existing algorithm which helps in insulin administration can be placed either within WA computations or within the data management components of the proposed architecture [11].

Secondly, we wanted to draw attention of healthcare professionals to the fact that current pumps do not generate sufficient data for creating a more natural administration of insulin. Current software solutions are determined by pump manufacturers, which does not necessarily mean that we can use them outside their operating environments.

There are two pathways from this research.

Firstly, we need to have a dialog with medical, healthcare professionals and pump manufacturers and decide which data from Table 1 must be present in computations for an AP. It is important that we address all three columns of data from Table 1 because we cannot see how else we can move towards an AP if we do not use data essential in the functioning of a natural pancreas.

Secondly, to create a full-scale application, we need to work with teams of healthcare professionals and software developers, which could determine the exact calculations performed by SQL persistence and OWL reasoning. The reusability of the model from Figure 3 guarantees that we can change or improve these calculations and reasoning, according to the data we collect from new devices, whenever required.

We believe that the data model from Table 1 and software architecture from Figure 3 are not difficult to convert into an Android App or to create a new Web Application run by patients. However, without thinking about new types of "pumps" which collect different data, we would not be able to use big Data Technologies, Analytics, Semantics stored in OWL and reason upon them. Patients would still be dependent on scheduled visits to their healthcare advisers to understand how successful their insulin administration is. Insulin administration will not be very similar to our natural organ.

The only way forward is interdisciplinary and collaborative research from healthcare professionals, patients, sensor/pump manufacturers and software developers. It appears that currently this collaboration does not exist.

\section{References}

[1] American Diabetes Association, "Diagnosis and Classification of Diabetes Mellitus", Diabetes Care, vol. 33, no. Suppl 1, 2010, pp. S62-S69.

[2] S. O. Bamgbose, X. Li, and L. Qian, "Closed loop control of blood glucose level with neural network predictor for diabetic patients", in 2017 IEEE 19th International Conference on e-Health Networking, Applications and Services (Healthcom), 2017, pp. 1-6.

[3] K. D. Barnard, K. K. Hood, J. Weissberg-Benchell, C. Aldred, N. Oliver, and L. Laffel, "Psychosocial Assessment of Artificial Pancreas (AP): Commentary and Review of Existing Measures and Their Applicability in AP Research", Diabetes Technology \& Therapeutics, vol. 17, no. 4, 2015. pp. 295-300.

[4] R. C. Bhake, J. A. Leendertz, A. C. E .Linthorst \& S. L. Lightman, "Continuous measurement of free cortisol profiles", Endocrine Abstracts 31, 2013, p. 324. 
[5] H. Blauw, P. Keith-Hynes, R. Koops, and J. H. DeVries, "A Review of Safety and Design Requirements of the Artificial Pancreas", Annals of Biomedical Engineering, vol. 44, no. 11, 2016, pp. 3158-3172

[6] D. Boiroux, T. B. Aradóttir, K. Nørgaard, N. K. Poulsen, H. Madsen, and J. B. Jørgensen, "An Adaptive Nonlinear Basal-Bolus Calculator for Patients With Type 1 Diabetes", Journal of Diabetes Science and Technology, vol. 11, no. 1, 2016, pp. 29-36.

[7] C. W. Chia, and C. D. Saudek, "Glucose sensors: Toward closed loop insulin delivery", Endocrinology and Metabolism Clinics of North America, vol. 33, no. 1, 2004, p. 21.

[8] D. Dawoud, R. O’Mahony, D. Wonderling, J. Cobb, B. Higgins, and S. A. Amiel, "Basal Insulin Regimens for Adults with Type 1 Diabetes Mellitus: A Systematic Review and Network Meta-Analysis," Value in Health, vol. 21, no. 2, 2018, pp. 176-184.

[9] A. E. Fathi, M. R. Smaoui, V. Gingras, B. Boulet, and A. Haidar, "The Artificial Pancreas and Meal Control: An Overview of Postprandial Glucose Regulation in Type 1 Diabetes", IEEE Control Systems, vol. 38, no. 1, 2018, pp. 67-85.

[10] R. Juric, "Could Semantic Web Technologies Create New Computational Models Outside Semantic Web", in Proceedings of the $21^{\text {st }}$ SDPS conference, Florida, 2016.

[11] R. Juric, and I. Kim, "Software Architectures for Smart Applications which Merge Ontological Reasoning with Big Data Analytics, in Proceedings of the $22^{\text {nd }}$ SDPS conference, Alabama, 2017.

[12] P. Keith-Hynes, B. Mize, A. Robert, and J. Place, "The Diabetes Assistant: A Smartphone-Based System for Real-Time Control of Blood Glucose", Electronics, vol. 3, no. $4,2014$.

[13] C. Lazaro, E. Oruklu, M. Sevil, K. Turksoy, and A. Cinar, "Implementation of an artificial pancreas system on a mobile device", in 2016 IEEE International Conference on Electro Information Technology (EIT), North Dakota, 2016, pp. 0642-0647.

[14] K. Løvås and E. Husebye, Endokrinologi - En medisinsk lærebok, 1 ed. Norway: Gyldendal, 2013.

[15] Medtronic MiniMed Inc, "User guide Medtronic MiniMed 640G," ed, 2015.

[16] "Nasjonal Diabetesplan 2017-202“" Available: https://www.regjeringen.no/no/dokumenter/nasjonaldiabetesplan-2017-2021/id2582010/?q=diabetes

[17] L. Ortmann, D. Shi, E. Dassau, F. J. Doyle, S. Leonhardt, and B. J. E. Misgeld, "Gaussian process-based model predictive control of blood glucose for patients with type 1 diabetes mellitus", in Proceedings 11th Asian Control Conference, Australia, 2017, pp. 1092-1097.

[18] N. Paulmann, M. Grohmann, JP. Voigt, B. Bert, J. Vowinckel, M. Bader, M. Skelin, M. Jevsek, H. Fink, M. Rupnik, and DJ. Walther, " Intracellular serotonin modulates insulin secretion from pancreatic beta-cells by protein serotonylation", in PLoS Bilogy, Oct, 7, 2009.

[19] P. E. Ross, "Diabetes has a new enemy: Robopancreas," IEEE Spectrum, vol. 52, no. 6, 2015, pp. 40-44.

[20] S. Samadi, K. Turksoy, I. Hajizadeh, J. Feng, M. Sevil, and A. Cinar, "Meal Detection and Carbohydrate
Estimation Using Continuous Glucose Sensor Data", IEEE Journal of Biomedical and Health Informatics, vol. 21, no. 3, 2017, pp. 619-627.

[21] A. Siebenhofer, J. Plank, A. Berghold, K. Horvath, P. Sawicki, P. Beck, and T. Pieber, "Meta-analysis of shortacting insulin analogues in adult patients with type 1 diabetes: continuous subcutaneous insulin infusion versus injection therapy", Diabetologia, vol. 47, no. 11, 2004, pp. 1895-1905.

[22] A. Skafjeld and M. Graue, “ Diabetes -

Forebygging, oppfølging, behandling“, 1. ed. Norway: Akribe, 2011.

[23] M. Tarabi, and R. Juric, "Software Architectures for Smart Applications in The Management Of Chronic Diseases: A Case Study of Reversibility of Diabetes 2, in Proceedings of the $51^{\text {st }}$ HICSS Conference, Hawaii, 2018.

[24] M. Tarabi, R. Juric, and S. Larsen, "Ontological Model for Building Java Applications in Personalised Healthcare: A Study of Reversibility Of Diabetes 2, in Proceedings of the $22^{\text {nd }}$ SDPS conference, Alabama, 2017.

[25] C. Toffanin, R. Visentin, M. Messori, F. D. Palma, L. Magni, and C. Cobelli, "Toward a Run-to-Run Adaptive Artificial Pancreas: In Silico Results", IEEE Transactions on Biomedical Engineering, vol. 65, no. 3, 2018, pp. 479488.

[26] S. Trevitt, S. Simpson, and A. Wood, "Artificial Pancreas Device Systems for the Closed-Loop Control of Type 1 Diabetes: What Systems Are in Development?", Journal of Diabetes Science and Technology, vol. 10, no. 3, 2016, pp. 714-723.

[27] K. Turksoy, E. Littlejohn, and A. Cinar, "Multimodule, Multivariable Artificial Pancreas for Patients with Type 1 Diabetes: Regulating Glucose Concentration Under Challenging Conditions," IEEE Control Systems, vol. 38, no. 1, 2018, pp. 105-124.

[28] K. Turksoy, S. Samadi, J. Feng, E. Littlejohn, L. Quinn, and A. Cinar, "Meal Detection in Patients With Type 1 Diabetes: A New Module for the Multivariable Adaptive Artificial Pancreas Control System", IEEE Journal of Biomedical and Health Informatics, vol. 20, no. 1, 2016, pp. 47-54.

[29] M. Waite, C. Martin, E. Franklin, D. Duce, and R. Harrison, "Human Factors and Data Logging Processes With the Use of Advanced Technology for Adults With Type 1 Diabetes: Systematic Integrative Review", (in eng), JMIR Human Factors, Review article vol. 5, no. 1, 2018.

[30] Y. Wang, E. Dassau, and F. J. Doyle, "ClosedLoop Control of Artificial Pancreatic beta-Cell in T1DM Using Model Predictive Iterative Learning Control", IEEE Transactions on Biomedical Engineering, vol. 57, no. 2, 2010, pp. 211-219.

[31] World Health Organization, "Global Report on Diabetes", Online, Online report, 2016, Available: http://www.who.int/diabetes/publications/grd-2016/en/.

[32] S. Zavitsanou, A. Mantalaris, M. C. Georgiadis, and E. N. Pistikopoulos, "In Silico Closed-Loop Control, Validation Studies for Optimal Insulin Delivery in Type 1 Diabetes", IEEE Transactions on Biomedical Engineering, vol. 62 , no. 10, 2015, pp. 2369-2378. 